\title{
IKERLANAK
}

\section{INCOME RISK OF EU COAL-FIRED POWER PLANTS AFTER KYOTO}

by

Luis M. Abadie and José M. Chamorro

2008

Working Paper Series: IL. 33/08

Departamento de Fundamentos del Análisis Económico I

Ekonomi Analisiaren Oinarriak I Saila

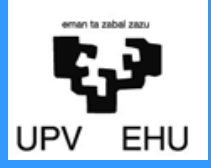

University of the Basque Country 


\title{
Income risk of EU coal-fired power plants after Kyoto
}

\author{
Luis M. Abadie \\ Bilbao Bizkaia Kutxa \\ Gran Vía, 30 \\ 48009 Bilbao, Spain \\ Tel +34-607408748 \\ Fax +34-944017996 \\ E-mail: imabadie@euskalnet.net \\ José M. Chamorro * \\ University of the Basque Country \\ Dpt. Fundamentos del Análisis Económico I \\ Av. Lehendakari Aguirre, 83 \\ 48015 Bilbao, Spain \\ Tel. +34-946013769 \\ Fax +34-946013891 \\ E-mail: jm.chamorro@ehu.es
}

October 10th 2008

\begin{abstract}
Coal-fired power plants may enjoy a significant advantage relative to gas plants in terms of cheaper fuel cost. Still, this advantage may erode or even turn into disadvantage depending on CO2 emission allowance price. This price will presumably rise in both the Kyoto Protocol commitment period (2008-2012) and the first post-Kyoto years. Thus, in a carbonconstrained environment, coal plants face financial risks arising in their profit margins, which in turn hinge on their so-called 'clean dark spread'. These risks are further reinforced when the price of the output electricity is determined by natural gas-fired plants' marginal costs, which differ from coal plants' costs.

We aim to assess the risks in coal plants' margins. We adopt parameter values estimated from empirical data. These in turn are derived from natural gas and electricity markets alongside the EU ETS market where emission allowances are traded. Monte Carlo simulation allows to compute the expected value and risk profile of coal-based electricity generation. We focus on the clean dark spread in both time periods under different
\end{abstract}


future scenarios in the allowance market. Specifically, bottom $5 \%$ and $10 \%$ percentiles are derived. According to our results, certain future paths of the allowance price may impose significant risks on the clean dark spread obtained by coal plants.

Keywords: Clean dark spread, clean spark spread, EU Emissions Trading Scheme, Monte Carlo.

JEL Codes: C6; E2; D8, G3.

Acknowledgement 1 A former version of this paper titled "Carbon price risk and the clean dark spread" has been presented at University of the Basque Country and the 3rd Workshop on Energy and Environmental Economics: Climate Change Policies after 2012", held at A Toxa (Spain). We thank seminar participants, and particularly Cruz A. Echevarría (UPVEHU, Bilbao) and Pedro Linares (Universidad Pontificia de Comillas, Madrid), for their valuable suggestions. Yet any error remains our own.

* Corresponding author.

\section{Introduction}

One of the main and most obvious consequences of electricity market liberalization is the fact that now utilities provide electricity at a variable price (as opposed to a fixed rate as in the traditional regulated framework). In addition, as integration gathers pace and affects fuel markets (such as coal or natural gas), input costs also become volatile. In a deregulated market, presumably only assets that enjoy a positive difference between the price of electricity and the price of a particular fuel used to generate it under prevailing market conditions will be operated. It is this spread that determines the economic value of a generation asset that can be used to transform the input fuel into output electricity. Thus, power operators pay close attention to the dark and spark spreads; Alberola et al. [2]. The dark spread represents the theoretical profit that a coal-fired power plant makes from selling a unit of electricity having purchased the fuel required to produce that unit of electricity. Similarly, the spark spread refers to the equivalent for natural gas-fired power plants. (This idea is analogous to the concept of the "crack spread" used in the oil/refining industry; Deng et al. [6]. It refers to the prices of crude oil and refined products like diesel or gasoline.)

On the other hand, within the EU Emissions Trading Scheme (ETS), the price of emission allowances can affect the cash flows of a power plant during its entire lifetime. Upon the introduction of carbon costs, the former spreads must be corrected by the allowance price. They become, respectively, the clean dark spread and the clean spark spread. As is well known, the option to switch from coal to natural gas in its inputs represents an abatement opportunity for a utility to reduce $\mathrm{CO} 2$ emissions in the short term. Therefore, the equilibrium between these spreads sets the carbon price above which it becomes profitable for an electricity producer to switch from coal to natural gas; Laurikka and Koljonen [14]. Conversely, as long as the carbon price remains below this switching price, coal plants are more profitable than gas plants, even after taking carbon costs 
into account. These indicators help to determine the preferred fuel used for power generation. Final consumers are also affected since allowance prices will presumably be passed on to electricity prices (either fully or in part).

This paper can be of interest also to U.S. utilities. In the EIA [8] reference case, coal-fired power plants continue to be the dominant source of electricity generation through 2030. Thus, coal share generation increases from $49 \%$ to $54 \%$, and coal plants account for $40 \%$ of total capacity additions from 2006 to 2030. However, technology choices for new plants and utilization of existing capacity are affected by relative fuel costs and changes in environmental policies. Right now, there is no federal cap-and-trade mechanism to control $\mathrm{CO}_{2}$ emissions. But a few multi-state or regional schemes are on the way, and federal legislation can eventually be established well before the next coal plants reach the end of their useful lives. If so, failure now to account properly for uncertain emission costs in investment decisions can press utilities into a rush for coal only to get their fingers burnt in the longer term. Indeed, many utilities are beginning to assess the potential cost of future carbon regulations within their resource plans. Following Barbose et al. [4], the mere prospect of that regulation is already having an impact on utilities' decision-making and resource choices.

Several papers address the valuation of electricity generating assets assuming separate dynamics for input and output prices. Hlouskova et al. [10] consider a coal-based electricity producing turbine in a liberalized market. They adopt a discrete-time model for the electricity spot price, which is a first-order autoregressive process with time-varying mean and a jump component. Coal price, instead, is assumed constant. Focusing on the relevant spot market for electricity in Germany, they value a typical electricity generating turbine alongside its optimal schedule. The (multi-stage) decision problem is solved by Monte Carlo simulation in conjunction with backward dynamic programming. Besides, the spot price model is used to simulate possible price paths for their sample period. The simulated profit-loss distribution represents the risk profile of the plant. This information can be used to integrate physical production assets with financial contracts for the purpose of enterprise-wide risk management.

Deng et al. [6] value both generation and transmission assets starting from futures contracts for electricity and the relevant fuel (e.g., natural gas). First they consider the case in which the futures price processes of electricity and gas for the relevant maturities follow geometric Brownian motions. Then they assume specific mean-reverting processes. Later on, Deng [5] extends the former model by adopting a more realistic electricity spot price model which explicitly takes into account jumps and spikes. Yet this process continues to be a onefactor model and as such has its drawbacks in matching the correlation structure of electricity forward prices with different maturities.

Laurikka [13] addresses the valuation of power technologies within an emissions trading scheme. He presents a simulation model in an operating environment with multiple exogenous and one-factor mean-reverting stochastic prices (among them, electricity, emission allowance, coal, natural gas). Laurikka and Koljonen [14] consider a similar environment; unlike the previous work, they 
consider two simple mean-reverting stochastic variables (the price of electricity and the emission allowance) and two deterministic variables (fuel price and the number of free allowances). Again, they adopt the Monte Carlo approach for plant valuation purposes.

Näsäkkälä and Fleten [15] follow a different approach. They take the viewpoint of an investor holding a license to build a gas-fired power plant. But they directly model the spark spread as a sum of short-term deviations (assumed to follow a simple mean-reverting process) and equilibrium price (assumed to follow a geometric Brownian motion). One advantage is that, as electricity and gas are often used for the same purposes (such as heating or cooling), their seasonal variations have similar phases and thus the seasonality decays from the spark spread. Also, it enables a more thorough and intuitive description of the investment decision process. As they point out, though, when using the spark spread process some information about its uncertainty structure may be lost compared to models with separate processes for input and output. However, after developing the two approaches, their results indicate that, with their data, the difference is small.

The purpose of this paper is to assess the risks in coal plants' earnings. Following a similar approach to Näsäkkälä and Fleten [15], we adopt the viewpoint of a utility which is operating a coal-fired power plant with a long useful life ahead (it is similar for an investor holding a license to build a coal-fired plant). Assuming it operates under the EU ETS, from the outset we naturally focus on the clean dark spread. Arguably, the prices of coal, natural gas, carbon and electricity are governed by stochastic processes. However, we do not propose separate dynamics for each of them. Somehow we blend the two approaches mentioned above. We start from the notion of the clean dark spread. But, instead of directly modelling it, we decompose it into three separate elements with simple interpretations. Then, we assume a specific continuous-time stochastic process for each of them.

First, we note that electricity price typically arises from supply and demand schedules. Thus, electricity is sold at the price requested by the marginal generating unit in the matching process. The cost of fuel composes most of the marginal cost of electricity. The structure of the merit order of suppliers may vary significantly from one country to another. Usually the marginal units are natural gas-fired plants; consequently the price of electricity is determined by the margin of this kind of technology, namely the clean spark spread (plus, possibly, other items as operating and maintenance costs, etc.). (Because gas-fired turbines produce relatively little pollution, and are quick and easy to start and ramp up, they are often the technology of choice for load following and peak generation; Douglas and Popova [7].) Yet the rest of the generating fleet in general, and coal-fired plants in particular, show a different pattern of fuel costs, emission costs, and the like. Under these circumstances, gas plants are better suited than coal plants to offset, at least partially, the risk in the cost side with the risk in the revenue side. Indeed, base load generators face their greatest risk of loss when prices and consumption fall, largely because they incur significant costs when they must shut down and restart base load power plants. Therefore 
their margins face a greater risk; note, though, that this does not necessarily mean less expected profits.

The first element in our decomposition of the clean dark spread is thus the clean spark spread. Similarly, the second one is also a composite item, namely the fuel price gap between coal and gas (per megawatt hour generated); the reason for doing so is explained below. But we do not further decompose these variables into other, more fundamental ones (e.g. the prices of electricity, gas, and so on); we directly model them as specific one-factor mean-reverting processes. As for the third element, the price of carbon dioxide, we assume it follows a standard geometric Brownian motion, and is treated as such in isolation. This may be a good compromise between information loss and intuitive explanation.

We estimate the three stochastic processes from actual market data. Thus we get numerical values of the underlying parameters. We then run a number of simulations for each of these three components. The whole time horizon comprises ten years, which span the Kyoto Protocol's commitment period (200812) and the next five-year period 2013-17. This time horizon is subdivided into many shorter time steps. At each step in a given simulation the resulting clean dark spread is derived; it is measured in euros per megawatt hour. Since this sum is received for one megawatt hour produced, income in that step equals the spread times the number of megawatts hour generated over that step. (The amount earned over any one time step may be interpreted as the sum that is contributed to the utility's income statement in that step.) Last, we just add up all the step contributions until the end of the time horizon considered. Thus we get an estimate of the cumulative income over the two five-year periods for a given simulation. Following this process a number of times allows us to assess not only the expected earnings but also the risk profiles. We note that there is no discounting in the valuation process since this is not deemed essential to the objective of the paper. According to our results, the earnings do remain positive over the commitment period. This no longer holds necessarily, though, in the immediate post-Kyoto period.

The remainder of our paper is organized as follows. In Section 2 we introduce some preliminaries about clean spreads along with the technical parameter values that will be used below. In Section 3 we explain the three stochastic processes that the aforementioned variables are assumed to follow. We describe our sample data and also the numerical estimates of the relevant parameters. In Section 4 we show the main results of our simulations regarding coal-based power plants under the EU ETS. Section 5 concludes.

\section{Some preliminaries}

\subsection{Conceptual framework}

Two different spreads can be defined. First, for a gas-fired plant the clean spark spread (CSS) is: 


$$
C S S=P_{E}-\frac{P_{G}}{E_{G}}-P_{C O_{2}} I_{G},
$$

where $P_{E}$ denotes electricity price $(€ / M W h), P_{G}$ is the price of natural gas $(€ / M W h),{ }^{1} E_{G}$ is the net thermal efficiency of a gas-fired plant, and $P_{\mathrm{CO}_{2}}$ is the price of a EU emission allowance $\left(€ / t C \mathrm{O}_{2}\right)$. Last, $I_{G}$ stands for the emission intensity of the plant $\left(t \mathrm{CO}_{2} / \mathrm{MWh}\right)$; this in turn depends upon the net thermal efficiency of each gas-fired plant.

According to IPCC [11], a plant burning natural gas has an emissions factor of $56.1 \mathrm{kgCO}_{2} / G J .^{2}$ Since under $100 \%$ efficiency conditions $3.6 \mathrm{GJ}$ would be consumed per megawatt hour, we get:

$$
I_{G}=\frac{0.20196}{E_{G}} \frac{t C O_{2}}{M W h} .
$$

Thus the complete formula for the CSS is:

$$
C S S=P_{E}-\frac{1}{E_{G}}\left(P_{G}+0.20196 \times P_{C O_{2}}\right) .
$$

Similarly, following IPCC [11] a plant burning bituminous coal has an emission factor of $94.6 \mathrm{kgCO}_{2} / \mathrm{GJ}$ under $100 \%$ efficiency conditions; then:

$$
I_{C}=\frac{0.34056}{E_{C}} \frac{t C O_{2}}{M W h} .
$$

Thus the complete formula for the Clean Dark Spread (CDS) is:

$$
C D S=P_{E}-\frac{1}{E_{C}}\left(P_{C}+0.34056 \times P_{C O_{2}}\right),
$$

where $P_{C}$ is the price of coal $(€ / M W h)$, and $E_{C}$ denotes the net thermal efficiency of a coal-fired plant.

We can solve for $P_{E}$ in Eq. (3) and then substitute into Eq. (5), thus linking the two spreads. This yields:

$$
C D S=C S S+\frac{1}{E_{G}}\left(P_{G}+0.20196 \times P_{C O_{2}}\right)-\frac{1}{E_{C}}\left(P_{C}+0.34056 \times P_{C O_{2}}\right) .
$$

Or, equivalently:

$$
C D S=C S S+\left(\frac{P_{G}}{E_{G}}-\frac{P_{C}}{E_{C}}\right)+\left(\frac{0.20196 \times P_{C O_{2}}}{E_{G}}-\frac{0.34056 \times P_{C O_{2}}}{E_{C}}\right) .
$$

With regard to the right-hand side, the first term represents the margin derived by natural gas plants. The second one is the fuel price gap (adjusted for

\footnotetext{
${ }^{1} 1 \mathrm{MWh}=3.412 \mathrm{mmBTU}$, and $1 \mathrm{mmBTU}=0.293083 \mathrm{MWh}$.

${ }^{2}$ This corresponds to $15.311 \mathrm{kgC} / G J$, since one ton of carbon is carried on 3.67 tons of $\mathrm{CO}_{2}$.
} 
relative efficiency rates). To the extent that this gap is positive, it is to the advantage of coal-fired plants. The last term, though, stands for the disadvantage of coal plants because of their higher carbon emissions, which is further aggravated by their lower efficiency levels.

Both spreads are equal $C S S=C D S$ when:

$$
\frac{P_{G}}{E_{G}}-\frac{P_{C}}{E_{C}}=\frac{0.34056 \times P_{C O_{2}}}{E_{C}}-\frac{0.20196 \times P_{C O_{2}}}{E_{G}},
$$

i.e., when the advantage in fuel price is wiped out by the higher emission costs.

For high enough allowance prices, CDS can reach a low value or even become negative. Specifically, we would get $C D S=0$ when:

$$
C S S+\frac{P_{G}}{E_{G}}-\frac{P_{C}}{E_{C}}=\frac{0.34056 \times P_{C O_{2}}}{E_{C}}-\frac{0.20196 \times P_{C O_{2}}}{E_{G}},
$$

i.e., when the higher costs of carbon permits offset not only the fuel price gap but the CSS as well.

Henceforth we adopt the typical case: $E_{G}=0.55$ and $E_{C}=0.40$. Then Eq. (2) implies overall emissions of $I_{G}=0.3672 t C O_{2} / M W h$ from a gas plant; and Eq. (4) implies overall emissions of $I_{C}=0.8514 t C O_{2} / M W h$ from a coal plant. In this case, $C D S=0$ when:

$$
C S S+\frac{P_{G}}{0.55}-\frac{P_{C}}{0.40}=\left(\frac{0.34056}{0.40}-\frac{0.20196}{0.55}\right) P_{C_{2}}=0.4842 P_{C O_{2}} .
$$

Hence, an increase of one euro in the allowance price reduces the CDS by 0.4842 $€ / M W h$.

\subsection{Sample data}

Our sample consists of four data sets: (1) daily electricity prices on PowerNext (France) from November 27th 2001 to February 6th 2008; (2) daily gas prices at Zeebrugge (Belgium) from April 4th 2001 to February 2nd 2008 as provided by Bloomberg; (3) daily spot carbon prices on the EU ETS from June 20th 2005 to January 28th 2008 as provided by BlueNext; (4) daily ARA coal one-month futures prices on the European Energy Exchange (Leipzig, Germany) from May 2nd 2006 to February 5th 2008. (In addition, prices of futures contracts on EU allowances from ICE have been used but only in Section 3.4 below, so we make no further reference to them here.) Sample prices come from markets that are geographically very close to each other. We focus on the time span over which the four price series are available, namely May-1-2006 to Jan-282008. We transform the daily series into weekly average prices. Thus, we have a complete data set over 92 weeks.

Figure 1 shows the time path of CSS and CDS series. Their main statistics appear in Table 1 . The average CDS value is significantly higher than the mean CSS. To a large extent this is due to low $\mathrm{CO}_{2}$ allowance prices; 
see Figure 2. Also, the advantage in fuel prices for a coal plant amounts to $8.8666 € / M W h$ on average. We can get a crude estimate of the carbon price necessary to have $C S S=C D S$; a rough computation would lead to $\widehat{P}_{\mathrm{CO}_{2}}=\frac{8.8666}{0.4842}=18.31 € / t C \mathrm{O}_{2}$. Similarly, Table 1 suggests that one would have $C D S=0$ for $\widetilde{P}_{\mathrm{CO}_{2}}=\frac{11.281+8.8666}{0.4842}=41.61 € / t C O_{2}$. Below we propose and then estimate the stochastic processes that will allow us to set long-term equilibrium values and provide more exact estimates.

\begin{tabular}{|llll|}
\hline \multicolumn{4}{|c|}{ Table 1. Basic statistics of CSS and CDS weekly series. } \\
\hline & $C S S$ & $C D S$ & $\frac{P_{G}}{0.55}-\frac{P_{C}}{0.40}$ \\
\hline Average $(€ / M W h)$ & 11.281 & 19.766 & 8.8666 \\
Median $(€ / M W h)$ & 8.2827 & 13.472 & 8.9994 \\
Minimum $(€ / M W h)$ & -17.338 & -11.047 & 0.83517 \\
Maximum $(€ / M W h)$ & 89.580 & 111.22 & 15.801 \\
Standard deviation & 15.717 & 18.839 & 4.0317 \\
Coeff. variation & 1.3932 & 0.95312 & 0.45470 \\
Skewness & 2.4997 & 2.4814 & -0.14031 \\
Excess kurtosis & 9.0810 & 7.6654 & -1.0235 \\
\hline
\end{tabular}

\section{Stochastic models and econometric analysis}

Following Eq. (6), first we assume specific continuous-time stochastic processes for each of the three items on the right-hand side. Then, we focus on their discrete-time counterparts. We estimate the coefficients on these regression equations with actual data from the markets involved. The relations between the coefficients in the discrete -time equations and the parameters in the continuoustime processes allow us to get numerical estimates of the latter from the former set of values. Subsequently we will use these parameter values in our MC simulations of the separate inputs to the CDS (and their sum).

\subsection{The Clean Spark Spread}

We adopt the simplest mean-reverting stochastic process (also known as an Ornstein-Uhlenbeck or O-U process):

$$
d S_{t}=k_{S}\left(S_{m}-S_{t}\right) d t+\sigma_{S} d W_{t}^{S}
$$

where $S_{t}$ denotes the value of the CSS at time $t$. This current value tends to the level of the CSS in the long term $S_{m}$ at a speed of reversion $k_{S}$. Besides, $\sigma_{S}$ is the instantaneous volatility of the CSS, and $d W_{t}^{S}$ stands for the increment to a standard Wiener process.

This model allows CSS to take on negative and positive values. It is shown in Appendix A that $S_{t}$ has a conditional mean

$$
E\left(S_{t}\right)=S_{0} e^{-k_{S}\left(t-t_{0}\right)}+S_{m}\left(1-e^{-k_{S}\left(t-t_{0}\right)}\right),
$$

which amounts to 


$$
E\left(S_{t+\Delta t}\right)=S_{t} e^{-k_{S} \Delta t}+S_{m}\left(1-e^{-k_{S} \Delta t}\right) .
$$

Also, the conditional variance is

$$
\operatorname{Var}\left(S_{t}\right)=\frac{\sigma_{S}^{2}}{2 k_{S}}\left[1-e^{-2 k_{S}\left(t-t_{0}\right)}\right]
$$

Since both mean and variance remain finite as $t \rightarrow \infty$, this process is stationary. ${ }^{3}$

Eq. (11) is the continuous-time version of a first-order autoregressive process in discrete time:

$$
S_{t+\Delta t}=S_{m}\left(1-e^{-k_{S} \Delta t}\right)+S_{t} e^{-k_{S} \Delta t}+\varepsilon_{t+\Delta t}^{S}=a_{S}+b_{S} S_{t}+\varepsilon_{t+\Delta t}^{S},
$$

where $\varepsilon_{t}^{S} \sim N\left(0, \sigma_{\varepsilon}^{S}\right)$, and the following notation holds:

$$
\begin{gathered}
a_{S} \equiv S_{m}\left(1-b_{S}\right) \Rightarrow S_{m}=\frac{a_{S}}{1-b_{S}}, \\
b_{S} \equiv e^{-k_{S} \Delta t} \Rightarrow k_{S}=-\frac{\ln b_{S}}{\Delta t} .
\end{gathered}
$$

This process for the CSS is stationary if $b_{S}<1$. Figure 3 shows the partial autocorrelation function of the CSS weekly series. Also, as shown in Appendix A:

$$
\begin{aligned}
\left(\sigma_{\varepsilon}^{S}\right)^{2} & =\frac{\sigma_{S}^{2}}{2 k_{S}}\left[1-e^{-2 k_{S} \Delta t}\right] \Rightarrow \\
& \Rightarrow \sigma_{S}^{2}=\frac{2 k_{S}\left(\sigma_{\varepsilon}^{S}\right)^{2}}{1-e^{-2 k_{S} \Delta t}}=\frac{2\left(\sigma_{\varepsilon}^{S}\right)^{2} \ln b_{S}}{\Delta t\left[b_{S}^{2}-1\right]}
\end{aligned}
$$

The last three equations will allow us to recover the continuous-time process parameters $\left(k_{S}, S_{m}, \sigma_{S}\right)$ upon estimation of the regression coefficients $\left(a_{S}, b_{S}\right)$ and the standard deviation of the regression residuals $\left(\sigma_{\varepsilon}^{S}\right)$.

\subsection{The adjusted price gap between coal and natural gas}

Now we turn to the second term in Eq. (6)'s right hand. Figure 4 shows the prices of natural gas and coal along with the resulting gap between fuel prices (in $€ / M W h$ produced) with the assumed efficiency rates. Again we adopt an Ornstein-Uhlenbeck process for the difference $P_{G} / 0.55-P_{C} / 0.40$. We have another $\mathrm{AR}(1)$ process as its counterpart in discrete time:

$$
C_{t+\Delta t}=C_{m}\left(1-e^{-k_{C} \Delta t}\right)+C_{t} e^{-k_{C} \Delta t}+\varepsilon_{t+\Delta t}^{C}=a_{C}+b_{C} C_{t}+\varepsilon_{t+\Delta t}^{C},
$$

\footnotetext{
${ }^{3}$ The CSS can be traced back to several individual prices of inputs and outputs. It may be worth noting that Eq. (11) can be a good approximation of this spread even if the underlying prices themselves do not follow O-U processes (Sodal, Koekebakker, and Aadland [16]).
} 
where $C_{t}$ denotes the price gap at time $t, C_{m}$ is the level of the gap in the long term, and $k_{C}$ stands for the speed of reversion. The remainder of the notation goes as before.

\subsection{Joint estimation of CSS and fuel price gap}

It is possible that both the CSS series and the price gap series are affected by common factors. This possibility suggests that separate estimation of each equation may not be suitable. Instead, we estimate them as a first-order vector autoregressive (VAR) process:

$$
\begin{gathered}
S_{t+\Delta t}=a_{S}+b_{S} S_{t}+\varepsilon_{t+\Delta t}^{S}, \\
C_{t+\Delta t}=a_{C}+b_{C} C_{t}+\varepsilon_{t+\Delta t}^{C} .
\end{gathered}
$$

The VAR(1) estimates of the coefficients in Eqs. (14) and (15) appear in the left hand of Table 2 ; they are statistically significant. From the series of residuals in both regressions we compute a correlation coefficient $\rho_{S, C}=0.2538$. The right hand of Table 2 shows the values of the underlying parameters in the stochastic processes $(\Delta t=1 / 52$, weekly observations) implied by these estimates.

\begin{tabular}{|c|c|c|c|c|}
\hline \multicolumn{5}{|c|}{ Table 2. Estimates of the CSS and fuel price gap series. } \\
\hline Coefficient & Estimate & $t$-statistic & Parameter & Estimate \\
\hline$\widehat{a_{S}}$ & 5.7196 & 3.400 & $k_{S}$ & 34.0747 \\
\hline$\widehat{b_{S}}$ & 0.5202 & 6.071 & $S_{m}$ & 11.9216 \\
\hline$\widehat{a_{C}}$ & 1.3285 & 2.629 & $\sigma_{S}$ & 127.65 \\
\hline$\widehat{b_{C}}$ & 0.8494 & 16.374 & $k_{C}$ & 8.5068 \\
\hline$\sigma_{\varepsilon}^{S}$ & 13.2060 & & $C_{m}$ & 8.8256 \\
\hline$\sigma_{\varepsilon}^{C}$ & 2.0458 & & $\sigma_{C}$ & 15.9928 \\
\hline
\end{tabular}

A low value of $\widehat{b_{S}}$ implies that the process for CSS quickly reverts back to its long-term mean, as can be seen in the high value of $k_{S}$. Figure 5 displays both the observed CSS values and those predicted by the model. Instead, $\widehat{b_{C}}$ is closer to one, which means that the fuel price gap behaves more like a random walk, as shown by a lower speed of reversion $k_{C}$.

Threshold allowance price for $C D S=C S S$ and $C D S=0$. Using the parameter values of $S_{m}$ and $C_{m}$ in a deterministic context yields:

a) $C D S=C S S$ for a carbon price $\widehat{P}_{C O_{2}}=\frac{C_{m}}{0.4842}=18.23 € / t C O_{2}$. Below this level, $C D S>C S S$ would hold and coal-based technology would be preferred.

b) $C D S=0$ for a carbon price $\widetilde{P}_{C_{2}}=\frac{C_{m}+S_{m}}{0.4842}=42.85 € / t C O_{2}$. Above this level, $C D S<0$ would hold and coal-fired plants would be shunned.

The results of the above analysis broadly agree with the empirical evidence in Table 1. There, the implied values were $18.31 € / t C O_{2}$ and $41.61 € / t C O_{2}$, respectively. 
Thus, if electricity price is set by gas-fired plants, a sizeable rise in the allowance price would be enough to offset the initial advantage in fuel price enjoyed by coal-fired plants. These stations could even cease to be profitable in the absence of carbon-capture units.

\subsection{Stochastic process for the emission allowance price}

During the Kyoto Protocol's commitment period (2008-2012) we assume that carbon price $A_{t}$ (in $€ / t C O_{2}$ ) follows a geometric Brownian motion (GBM):

$$
d A_{t}=\alpha A_{t} d t+\sigma_{A} A_{t} d W_{t}^{A} .
$$

Therefore, the expected value for the allowance price in the near future is:

$$
E\left(A_{t}\right)=A_{0} e^{\alpha t} \text { for } t<5 .
$$

At the end of this period we assume there will be a sudden jump $J$ in price, which would push the expected value upwards:

$$
\begin{aligned}
& t=5^{-}: E\left(A_{t}\right)=A_{0} e^{5 \alpha} \\
& t=5^{+}: E\left(A_{t}\right)=A_{0} e^{5 \alpha}+J
\end{aligned}
$$

From then on, we assume allowance scarcity is just right as an environmental policy measure and price evolves once again following a GBM:

$$
E\left(A_{t}\right)=A_{0} e^{\alpha t}+J e^{\alpha(t-5)} \text { for } t>5 .
$$

No further jumps are assumed in subsequent years for the sake of simplicity. Though environmental policy is conceivably expected to become stricter and push allowance prices to new heights at the end of this period, it is hard to foresee what will happen then. ${ }^{4}$

Estimation. The European Climate Exchange (ECX) manages the European Climate Exchange Financial Instruments (ECX CFI), which are traded at the London-based International Petroleum Exchange (later acquired by the Intercontinental Exchange, or ICE). Futures prices from ICE have been used here.

As this paper was developed, there were futures contracts for both the threeyear trial trading period (2005-2007) and the commitment period (2008-2012). Our sample starts on May 1st 2006 and closes on March 26th 2008. We thus have 495 daily prices for each of the five futures contracts maturing from Dec-08 to Dec-12.

\footnotetext{
${ }^{4}$ In January 2008 the European Commission presented a proposal to revise and strengthen the ETS. Some of the main changes are the following: (a) There will be one EU-wide cap on the number of emission allowances instead of 27 national caps. The annual cap will decrease along a linear trend line, which will continue beyond the end of the third trading period (2013-2020). (b) A much larger share of allowances will be auctioned instead of allocated free of charge. (c) Harmonised rules governing free allocation will be introduced.
} 
Parameter estimation for the commitment period is developed as follows. First, we focus on the days for which observed futures prices are available. Then we estimate the spot price on each day by means of cubic splines that pass along the observed quotes on those days; see Figure 6 for a particular day. This procedure is briefly described in Appendix B. Estimation results of the spot price and nearby futures prices are plotted in Figure 7.

According to Ito's Lemma, the transformed variable $X_{t} \equiv \ln A_{t}$ follows a stochastic process:

$$
d X_{t}=\left(\alpha-\frac{\sigma_{A}^{2}}{2}\right) d t+\sigma_{A} d W_{t}^{A}
$$

Hence, in discrete time:

$$
y_{t}=\Delta \ln A_{t}=\ln A_{t}-\ln A_{t-\Delta t}=\left(\alpha-\frac{\sigma_{A}^{2}}{2}\right) \Delta t+\sigma_{A} \sqrt{\Delta t} \epsilon_{t}^{3},
$$

where $\epsilon_{t}^{3}$ is a standard Gaussian white noise.

We carry out our estimation according to the maximum likelihood (ML) method:

$$
\arg \min _{\alpha, \sigma_{A}} \sum_{t=1}^{T}\left[-\frac{1}{2} \ln \left(\frac{\sigma_{A}^{2}}{\Delta t}\right)-\frac{1}{2} \frac{\left(y_{t}-\left(\alpha-\frac{\sigma_{A}^{2}}{2}\right) \Delta t\right)^{2}}{\sigma_{A}^{2} \Delta t}\right] .
$$

This method (conditioned on the first observation $A_{0}$ ) provides the estimators of the mean and variance of $y_{t}$. They are given by:

$$
\widehat{m}=\frac{1}{T} \sum_{t=1}^{T} y_{t}, \quad \widehat{s}^{2}=\frac{1}{T} \sum_{t=1}^{T}\left(y_{t}-\widehat{m}\right)^{2} .
$$

On this occasion, we use the whole series of (almost five hundred) daily returns. These equations yield: $\widehat{m}=0.00051248, \widehat{s}^{2}=0.00082893$.

The ML estimators of the $\operatorname{drift}(\widehat{\alpha})$ and volatility $\left(\widehat{\sigma}_{A}\right)$ parameters are derived from their relations to the mean and variance parameters. Note that both $\widehat{m}$ and $\widehat{s}^{2}$ are expressed in daily terms, whereas $\widehat{\alpha}$ and $\widehat{\sigma}_{A}^{2}$ are customarily expressed in yearly terms. We take the number of trading days over a year to be 250 . Therefore we have $\Delta t=1 / 250$, and ML estimation yields: $\widehat{\sigma}_{A}^{2}=$ $\widehat{s}^{2} / \Delta t=250 \widehat{s}^{2}=0.2072$. Hence we get a volatility estimate of $\widehat{\sigma}_{A}=0.4552$. In this respect, if instead we adopt moving windows of 50 observations, volatility estimates evolve as shown in Figure 8. A value (here assumed constant) of 0.4552 does not seem off the mark. ${ }^{5}$

Regarding the estimation of the drift, this would be:

$$
\widehat{\alpha}=\frac{\widehat{m}}{\Delta t}+\frac{\widehat{s}^{2}}{2 \Delta t}=250 \widehat{m}+125 \widehat{s}^{2}=0.2317 .
$$

\footnotetext{
${ }^{5}$ Also, Abadie and Chamorro [1] estimate $\sigma_{A}$ from a similar sample by applying the Kalman filter. The numerical value is 0.4683 .
} 
Thus, the drift parameter of the process for $X_{t}$ is: $\widehat{\alpha}-\widehat{\sigma}_{A}^{2} / 2=250 \widehat{m}=0.1281$.

According to Gourieroux and Jasiak [9], asymptotic variances are given by:

$$
V_{\text {asy }}\left(\widehat{\sigma}_{A}^{2}\right)=\frac{2 \sigma_{A}^{4}}{T}=0.00017529, \quad V_{\text {asy }}(\widehat{\alpha})=\frac{\sigma_{A}^{2}}{T \Delta t}+\frac{\sigma_{A}^{4}}{2 T}=0.10577 .
$$

While volatility can be estimated accurately, this does not hold for the drift. Its asymptotic variance converges only mildly as the number of observations increases because of the term $T \Delta t$ which, in our case, amounts to $490 / 250=$ 1.96; this is why it has fallen only to half $\widehat{\sigma}_{A}^{2}$. Consequently, in what follows we use the volatility estimate but not that of the drift; instead, we adopt a range of possible values $\alpha=\{-0.025,0.00,0.025,0.05,0.075,0.10\}$ and analyze their effect.

Last, residuals from regression Eqs. (14), (15), and (20) allow us to compute the correlation coefficients: $\rho_{S, A}=-0.0122, \rho_{C, A}=0.0994$.

\section{Monte Carlo simulation}

As stated earlier, our aim is to study value and risk involved in coal stations operating under the EU ETS after Kyoto Protocol's expiration. We accomplish this by means of simulation techniques. ${ }^{6}$

\subsection{The components of the CDS in isolation}

Correlated random variables are generated according to the scheme (see Appen$\operatorname{dix} \mathrm{C})$ :

$$
\begin{gathered}
S_{t+\Delta t}=S_{m}\left(1-e^{-k_{S} \Delta t}\right)+S_{t} e^{-k_{S} \Delta t}+\sigma_{S} \sqrt{\frac{1-e^{-2 k_{S} \Delta t}}{2 k_{S}}} \epsilon_{t}^{1} \\
C_{t+\Delta t}=C_{m}\left(1-e^{-k_{C} \Delta t}\right)+C_{t} e^{-k_{C} \Delta t}+\sigma_{C} \sqrt{\frac{1-e^{-2 k_{C} \Delta t}}{2 k_{C}}}\left[\epsilon_{t}^{1} \rho_{S, C}+\epsilon_{t}^{2} \sqrt{1-\rho_{S, C}^{2}}\right] \\
\ln A_{t+\Delta t}=\ln A_{t}+\left(\alpha-\frac{\sigma_{A}^{2}}{2}\right) \Delta t+\sigma_{A} \sqrt{\Delta t}\left[\epsilon_{t}^{1} \rho_{S, A}+\right. \\
\left.\quad+\epsilon_{t}^{2} \frac{\rho_{C, A}-\rho_{S, A} \rho_{S, C}}{\sqrt{1-\rho_{S, C}^{2}}}+\epsilon_{t}^{3} \sqrt{1-\rho_{S, A}^{2}-\frac{\left(\rho_{C, A}-\rho_{S, A} \rho_{S, C}\right)^{2}}{1-\rho_{S, C}^{2}}}\right]
\end{gathered}
$$

or

${ }^{6}$ Alesii [3] develops a similar approach and shows a numerical example in the context of shipping finance. 


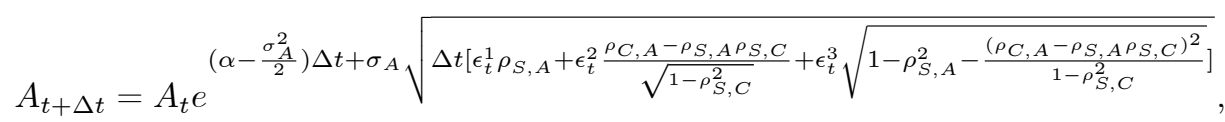

where $\epsilon_{t}^{1}, \epsilon_{t}^{2}$ and $\epsilon_{t}^{3}$ are standardized Gaussian white noises with zero correlation. The first expression above is derived after replacing $\sigma_{\varepsilon}^{S}$ in terms of $\sigma_{S}$. Similarly in the second expression. At the same time, if samples from a standardized bivariate normal distribution are required, an appropriate procedure is the one shown above, where $\rho_{S, C}, \rho_{S, A}$ and $\rho_{C, A}$ are the correlation coefficients between the variables in the multivariate distribution.

In order to get numerical estimates 40,000 sample paths for $S_{t}, C_{t}$ and $A_{t}$ are generated. Each path comprises 600 steps; this means five steps per month since the horizon considered is ten years, namely the five-year commitment period plus the next five years.

Starting values appear in Table 3; they have been computed above. As initial values of the CSS and the gap in fuel prices (in $€ / M W h$ ) we adopt the long-term equilibrium values. The initial allowance price is the one estimated on $03 / 26 / 2008$, the last day of the sample used to estimate this market's parameters.

\begin{tabular}{|llll|}
\hline \multicolumn{4}{|l|}{ Table 3. Initial parameter values of the MC simulation. } \\
\hline CSS & $S_{0}=S_{m}=11.9216$ & $k_{S}=34.0747$ & $\sigma_{S}=127.65$ \\
Fuel price gap & $C_{0}=C_{m}=8.8256$ & $k_{C}=8.5068$ & $\sigma_{C}=15.9928$ \\
Allowance price & $A_{0}=22.0923$ & - & $\sigma_{A}=0.4552$ \\
Correlation & $\rho_{S, C}=0.2538$ & $\rho_{S, A}=-0.0122$ & $\rho_{C, A}=0.0994$ \\
\hline
\end{tabular}

Computations are realized per unit of installed capacity (MW) and assume that the coal-fired plant operates $80 \%$ of the time on average (i.e. $8,760 \times 0.8=$ 7,008 hours over a year). (For any other percentage rate, computations would vary proportionately for each $M W$ of installed capacity.) Hence we assume a yearly production of 7,008 $M W h$ for each megawatt installed. Since 60 time steps are used per year, this implies that $116.8 M W h$ will be generated per step for each MW of capacity.

At the final time $t=10$, the distributions of the CSS and the fuel price gap are characterized by the statistics in Table 4 . The theoretical mean for $t=10$ amounts to the long-term level estimated before. The theoretical volatility for $t=10$ is computed according to the following formulas (see Appendix A):

$$
\sigma_{S, t=10}=\sigma_{S} \sqrt{\frac{1-e^{-20 k_{S}}}{2 k_{S}}}=15.4629, \quad \sigma_{C, t=10}=\sigma_{C} \sqrt{\frac{1-e^{-20 k_{C}}}{2 k_{C}}}=3.8066 .
$$

Table 4. Statistics of theoretical and simulated CSS and fuel price gap.

\begin{tabular}{|l|ll|ll|}
\hline & \multicolumn{2}{|c|}{ CSS } & \multicolumn{2}{c|}{ Fuel price gap } \\
\hline & Theoretical & Simulated & Theoretical & Simulated \\
Mean & 11.9216 & 11.8502 & 8.8256 & 8.8525 \\
Volatility & 15.4629 & 15.3780 & 3.8066 & 3.8673 \\
\hline
\end{tabular}


As for the allowance price, the final distribution at time $t=10$ of the allowance prices depends on the growth rate $\alpha$ used and the jump that would take place at the end of the commitment period. For example, in the case of $\alpha=0.05$ and a jump of 2.5 euros, the theoretical value of the mean would be:

$$
E\left(A_{t=10}\right)=A_{o} e^{0.50}+2.5 e^{0.25}=39.6341,
$$

as opposed to the mean from the 40,000 simulations of $40.143 € / t C \mathrm{O}_{2}$.

On the other hand, when $\alpha=0.05$ and the jump size is $0.0 €$, the theoretical value of volatility would be:

$$
\sigma_{A, t=10}=\sigma_{A} \sqrt{10}=1.4395 .
$$

This figure is very close to the value of 1.4409 derived from computing the standard deviation of the (natural $\log$ ) prices of the emission allowance at the end of the MC simulation. These similarities attest to the goodness of our sample paths.

\subsection{The overall CDS}

Tables 5-7 below show our results under different scenarios. We focus on average earnings as derived from CDS values in Table 5 along with the $5 \%$ and $10 \%$ percentiles in Tables 6 and 7. (We disregard other inputs to the plant's net final profit.)

As already mentioned, the ten-year horizon is partitioned into 600 time steps, or 60 steps per year. Also, we are assuming a yearly production of 7,008 $M W h$ for each megawatt of capacity installed; thus $116.8 M W h$ are generated over each step. The contribution of one step to the utility's economic results emanates from this output electricity and the accompanying CDS over this step. Aggregating over the first 300 time steps we compute the cumulative contribution of the plant to the firm's profits during the Kyoto period 20082012; similarly for the last 300 steps and the post-Kyoto period 2013-2017. No discounting is involved to better resemble what would show up in the utility's internal financial statements.

Look, for instance, at the Kyoto period under the assumption $\alpha=0.0 \%$ (first row in Table 5). When emission allowances are not expected to appreciate, the plant earns 352,000 euros over that time horizon (for megawatt installed). The output generated over this five-year period amounts to $7,008 \times 5=35,040$ $M W h$. Therefore the firm would get on average some $10.05 € / M W h .^{7}$ Next period (2013-17), if the expected drift rate remains at zero and there is no jump in allowance price, the average CDS remains almost the same, namely 348,000 euros (see next line in Table 5). (These values are not exactly equal since random samples are being generated in both cases and average values

\footnotetext{
${ }^{7}$ This is much lower than the average CDS of 19.76 that appears in Table 1 . This is partly due to low allowance prices during that sample period (Figure 2). According to our analysis, this is not what carbon market seems to suggest for the near future.
} 
may diverge slightly.) Obviously, if carbon price jumps upwards the CDS falls accordingly; similarly for changes in the value of the drift rate, $\alpha$.

\begin{tabular}{|cccccccc|}
\hline \multicolumn{5}{|c|}{ Table 5. Expected earnings per megawatt installed (thousand $€$ ) } \\
& $\alpha=-2.5 \%$ & $\alpha=0$ & $\alpha=2.5 \%$ & $\alpha=5 \%$ & $\alpha=7.5 \%$ & $\alpha=10 \%$ \\
\hline $2008-12$ & 374 & 352 & 327 & 300 & 271 & 239 \\
$2013-17$ & & & & & & \\
$\mathrm{~J}=0.0 €$ & 413 & 348 & 270 & 174 & 58 & -84 \\
$\mathrm{~J}=2.5 €$ & 373 & 306 & 224 & 126 & 6 & -140 \\
$\mathrm{~J}=5.0 €$ & 333 & 263 & 179 & 78 & -45 & -194 \\
$\mathrm{~J}=7.5 €$ & 293 & 221 & 134 & 29 & -97 & -249 \\
$\mathrm{~J}=10 €$ & 253 & 178 & 89 & -19 & -148 & -304 \\
\hline
\end{tabular}

\begin{tabular}{|lcccccc|}
\hline \multicolumn{6}{c|}{ Table 6. Earnings 10\% percentile (thousand $€$ ) in 2013-17. } \\
& $\alpha=-2.5 \%$ & $\alpha=0$ & $\alpha=2.5 \%$ & $\alpha=5 \%$ & $\alpha=7.5 \%$ & $\alpha=10 \%$ \\
\hline $\mathrm{J}=0.0 €$ & 17 & -126 & -300 & -511 & -770 & $-1,091$ \\
$\mathrm{~J}=2.5 €$ & -41 & -187 & -365 & -583 & -847 & $-1,166$ \\
$\mathrm{~J}=5.0 €$ & -98 & -249 & -431 & -652 & -922 & $-1,247$ \\
$\mathrm{~J}=7.5 €$ & -156 & -310 & -496 & -723 & -996 & $-1,328$ \\
$\mathrm{~J}=10 €$ & -216 & -374 & -564 & -797 & $-1,077$ & $-1,409$ \\
\hline
\end{tabular}

\begin{tabular}{|ccccccc|}
\hline \multicolumn{7}{c|}{ Table 7. Earnings 5\% percentile (thousand $€$ ) in 2013-17. } \\
& $\alpha=-2.5 \%$ & $\alpha=0$ & $\alpha=2.5 \%$ & $\alpha=5 \%$ & $\alpha=7.5 \%$ & $\alpha=10 \%$ \\
\hline $\mathrm{J}=0.0 €$ & -357 & -586 & -854 & $-1,187$ & $-1,596$ & $-2,096$ \\
$\mathrm{~J}=2.5 €$ & -423 & -652 & -930 & $-1,271$ & $-1,685$ & $-2,191$ \\
$\mathrm{~J}=5.0 €$ & -494 & -727 & $-1,011$ & $-1,355$ & $-1,776$ & $-2,290$ \\
$\mathrm{~J}=7.5 €$ & -562 & -800 & $-1,093$ & $-1,443$ & $-1,871$ & $-2,386$ \\
$\mathrm{~J}=10 €$ & -634 & -880 & $-1,176$ & $-1,534$ & $-1,965$ & $-2,492$ \\
\hline
\end{tabular}

It can be seen in Table 5 that, over the commitment period (2008-12), the expected average CDS takes on a positive value. Nonetheless, for values of the drift parameter $\alpha \geq 5 \%$, the expected CDS may turn to negative in the period 2013-17, the more so depending on the potential jump in allowance prices during the transition from one period to the next. These results are displayed in Figure 9.

Tables 6 and 7 show the Earnings at Risk $(E a R)$ of coal-fired plants in the 2013-17 phase. According to Table 6, in the absence of a jump and for $\alpha=5.0 \%$, there is a $10 \%$ of potential states in which losses would surpass 511,000 euros for each megawatt of capacity installed if electricity is produced under such circumstances. As shown in Table 7 , in the same scenario $(J=0.0$ $€$ and $\alpha=5.0 \%$ ), the losses would go beyond 1 million euros in the worst $5 \%$ of situations. See Figure 10. Presumably a coal-fired plant would not operate under such circumstances, unless other reasons imply so (signed guarantees or the like). (Nonetheless the figures may be taken as such so as to maintain the level basis for comparisons.) 


\section{CONCLUSIONS}

Present and future liabilities by EU utilities in relation to $\mathrm{CO} 2$ emissions create a setup previously unknown. Current legislation entails new costs and risks to the generating firms. Fortunately they are getting better gauged as markets develop and price series get longer.

We assume that electricity fetches a market price which is determined by natural gas combined-cycle plants operating as the marginal generating units. We further assume that the price gap between gas and coal follows a meanreverting process. Even though coal-fired plants enjoy an initial advantage in terms of cheap fuel for generating electricity, an increase in the price of the emission allowances may erode significantly their profit margins. Given that the future path of the allowances is hard to forecast (specially after 2012), we have designed potential scenarios depending on the drift rate of the allowance price and the jump size of the price at the end of the commitment period. Under these assumptions we have assessed the risks on the profit margins of coal plants.

Margins do remain positive over the Kyoto Protocol's commitment period. This no longer holds necessarily, though, in the immediate five years after the Protocol's expiration. Expected margins may switch to negative, or remain slightly positive but with a high risk of becoming negative in many cases. In such scenarios, this would lead to shut down the coal plant thus reducing the chances to recover the investment costs.

Realization of scenarios involving steep growth in the allowance price would be incompatible with investing in new coal plants. It could even trigger the abandonment of plants currently in operation but with relatively short useful lives as carbon prices climb ever higher. However, the decision to abandon a power plant may be affected by provisions in the National Allocation Plants concerning assigned allowances in the case of plant closures. Also, a high allowance price could be compatible with coal-based electricity generation if there are gains in efficiency rates and the plants are retrofitted with carbon capture and storage units, for example.

Similarly, in the case of developing countries still unaffected by the Kyoto Protocol, the signing and ensuing adaptation may put current investments in

this technology at risk. Consequently, the decision to undertake new investments should take this possibility into account.

\section{A Moments of a mean-reverting $\mathrm{O}-\mathrm{U}$ process}

We start from the stochastic model:

$$
d S_{t}=k\left(S_{m}-S_{t}\right) d t+\sigma_{t} d W_{t} .
$$

Next we adopt the notation in Kloeden and Platen [12]. The homogeneous equation is:

$$
\frac{d S_{t}}{S_{t}}=-k d t
$$


Therefore, its fundamental solution is $\Phi_{t, t_{0}}=e^{-k\left(t-t_{0}\right)}$ and making $Y_{t}=$ $\Phi_{t, t_{0}}^{-1} S_{t}=e^{k\left(t-t_{0}\right)} S_{t}$ derivatives can be computed:

$$
\frac{d Y_{t}}{d S_{t}}=e^{k\left(t-t_{0}\right)} ; \frac{d^{2} Y_{t}}{d S_{t}^{2}}=0 ; \frac{d Y_{t}}{d t}=k e^{k\left(t-t_{0}\right)} S_{t} .
$$

By Ito's Lemma:

$$
d Y_{t}=d\left(\Phi_{t, t_{0}}^{-1} S_{t}\right)=k S_{m} e^{k\left(t-t_{0}\right)} d t+e^{k\left(t-t_{0}\right)} \sigma_{t} d W_{t} ;
$$

hence we deduce that:

$$
S_{t}=S_{0} e^{-k\left(t-t_{0}\right)}+e^{-k\left(t-t_{0}\right)} k \int_{t_{0}}^{t} e^{k\left(s-t_{0}\right)} d s+e^{-k\left(t-t_{0}\right)} \sigma \int_{t_{0}}^{t} e^{k\left(s-t_{0}\right)} d W_{s}
$$

The first moment can be easily reached:

$$
E\left(S_{t}\right)=S_{0} e^{-k\left(t-t_{0}\right)}+S_{m}\left(1-e^{-k\left(t-t_{0}\right)}\right)
$$

therefore:

$$
E\left(S_{\infty}\right)=S_{m}
$$

The variance is given by:

$$
\operatorname{Var}\left(S_{t}\right)=e^{-2 k\left(t-t_{0}\right)} \sigma^{2} \int_{t_{0}}^{t} e^{2 k\left(s-t_{0}\right)} d s=\frac{\sigma^{2}}{2 k}\left[1-e^{-2 k\left(t-t_{0}\right)}\right] .
$$

\section{B Estimation of the cubic spline}

Each day we need to trace a curve that passes through all the points in the price/time space for which futures prices are available. For this purpose we use four pieces of curves of the form:

$$
\begin{aligned}
& y_{t}=a_{08} t^{3}+b_{08} t^{2}+c_{08} t+d_{08} \text { for } t_{08} \leqq t \leqq t_{09}, \\
& y_{t}=a_{09} t^{3}+b_{09} t^{2}+c_{09} t+d_{09} \text { for } t_{09} \leqq t \leqq t_{10}, \\
& y_{t}=a_{10} t^{3}+b_{10} t^{2}+c_{10} t+d_{10} \text { for } t_{10} \leqq t \leqq t_{11}, \\
& y_{t}=a_{11} t^{3}+b_{11} t^{2}+c_{11} t+d_{11} \text { for } t_{11} \leqq t \leqq t_{12},
\end{aligned}
$$

where $t_{08}, t_{09}, t_{10}, t_{11}$ and $t_{12}$ denote the time of maturity of the futures contracts from December-08 to December-12. For the sake of simplicity, and without loss of generality, we take $t_{08}=0$; then $t_{09}=1, t_{10}=2, t_{11}=3$ and $t_{12}=4$.

Since there are 16 parameters to estimate we need the same number of restrictions to get a unique solution: 
a) The pieces of the cubic functions must reach the points actually observed; this provides eight equations.

b) The first derivatives in $t_{09}, t_{10}$ and $t_{11}$ must coincide; three equations.

c) Coincidence of the second derivatives in $t_{09}, t_{10}$ and $t_{11}$; three equations.

d) The last two conditions have been chosen so as to get a natural spline; this requires that second derivatives at the initial and final points take on a value of zero.

The first cubic on each day, substituting the time to maturity of the Dec-08 futures contract with negative sign, allows to get an estimate for the spot price of the emission allowance on that day $A_{0}$. This can be seen in Figure 6.

\section{Generation of mean-reverting correlated vari- ables}

Let $e_{1}, e_{2}$, and $e_{3}$ be standard normal deviates. Random samples of correlated variables can be generated as follows:

$$
\begin{aligned}
& x_{1}=f_{11} e_{1}, \\
& x_{2}=f_{21} e_{1}+f_{22} e_{2}, \\
& x_{3}=f_{31} e_{1}+f_{32} e_{2}+f_{33} e_{3},
\end{aligned}
$$

if $\operatorname{Cov}\left(x_{1}, x_{2}\right)=\rho_{12}, \operatorname{Cov}\left(x_{1}, x_{3}\right)=\rho_{13}$, and $\operatorname{Cov}\left(x_{2}, x_{3}\right)=\rho_{23}$.

Random deviates with this correlation structure must satisfy:

$$
\begin{aligned}
E\left(x_{1}^{2}\right) & =1 \Longrightarrow f_{11}=1 \\
E\left(x_{1} x_{2}\right) & =\rho_{12} \Longrightarrow f_{21}=\rho_{12}, \\
E\left(x_{2}^{2}\right) & =1=f_{21}^{2}+f_{22}^{2} \Longrightarrow f_{22}=\sqrt{1-\rho_{12}^{2}} \\
E\left(x_{1} x_{3}\right) & =\rho_{13}=f_{11} f_{31} \Longrightarrow f_{31}=\rho_{13}, \\
E\left(x_{2} x_{3}\right) & =\rho_{23}=f_{21} f_{31}+f_{22} f_{32} \Longrightarrow f_{32}=\frac{\rho_{23}-\rho_{12} \rho_{13}}{\sqrt{1-\rho_{12}^{2}}}, \\
E\left(x_{3}^{2}\right) & =1=f_{31}^{2}+f_{32}^{2}+f_{33}^{2}=\rho_{13}^{2}+\frac{\left(\rho_{23}-\rho_{12} \rho_{13}\right)^{2}}{1-\rho_{12}^{2}}+f_{33}^{2} \Longrightarrow f_{33}=\sqrt{1-\rho_{13}^{2}-\frac{\left(\rho_{23}-\rho_{12} \rho_{13}\right)^{2}}{1-\rho_{12}^{2}}}
\end{aligned}
$$

Henceforth:

$$
\begin{aligned}
& x_{1}=e_{1}, \\
& x_{2}=e_{1} \rho_{12}+e_{2} \sqrt{1-\rho_{12}^{2}}, \\
& x_{3}=e_{1} \rho_{13}+e_{2} \frac{\rho_{23}-\rho_{12} \rho_{13}}{\sqrt{1-\rho_{12}^{2}}}+e_{3} \sqrt{1-\rho_{13}^{2}-\frac{\left(\rho_{23}-\rho_{12} \rho_{13}\right)^{2}}{1-\rho_{12}^{2}}} .
\end{aligned}
$$




\section{References}

[1] Abadie L.M. and Chamorro J.M.: "European CO2 prices and carbon capture investments". Energy Economics 30, 1850-1881, 2008.

[2] Alberola E., Chevallier J. and Chèze B.: "Price drivers and structural breaks in European carbon prices 2005-2007". Energy Policy 36, 787-797, 2008.

[3] Alesii G.: "VaR in real options analysis". Review of Financial Economics 14, 189-208, 2005.

[4] Barbose G., Wiser R., Phadke A., and Goldman C.: "Managing carbon regulatory risk in utility resource planning: Current practices in the Western United States". Energy Policy 36, 3300-3311, 2008.

[5] Deng S-J.: "Valuation of investment and opportunity-to-invest in power generation assets with spikes in electricity price". Managerial Finance 31(6): 95-115, 2005.

[6] Deng S-J., Johnson B. and Sogomonian A.: "Exotic electricity options and the valuation of electricity generation and transmission assets". Decision Support Systems 30: 383-392, 2001.

[7] Douglas S. and Popova J.: "Storage and the electricity forward premium". Energy Economics (2008), doi:10.1016/j.eneco.2007.12.013.

[8] Energy Information Administration: Annual Energy Outlook 2008.

[9] Gourieroux C. and Jasiak J.: Financial Econometrics. Princeton Series in Finance, 2001.

[10] Hlouskova J., Kossmeier S., Obersteiner M. and Schnabl A.: "Real options and the value of generation capacity in the German electricity market". Review of Financial Economics 14, 297-310, 2005.

[11] IPCC: Guidelines for National Greenhouse Gas Inventories, 2006.

[12] Kloeden P.E. and Platen E.: "Numerical Solution of Stochastic Differential Equations". Springer, 1992.

[13] Laurikka H., 2006. Option value of gasification technology within an emission trading scheme. Energy Policy 34, 3916-3928.

[14] Laurikka H., Koljonen T., 2006. Emissions trading and investment decisions in the power sector: a case study in Finland. Energy Policy 34, 1063-1074.

[15] Näsäkkälä E. and Fleten S-E.: "Flexibility and technology choice in gas fired power plant investments". Review of Financial Economics 14: 371393, 2005. 
[16] Sodal S., Koekebakker S., and Aadland R.: "Market switching in shipping: A real option model applied to the valuation of combination carriers". Review of Financial Economics 17, 183-203, 2008.

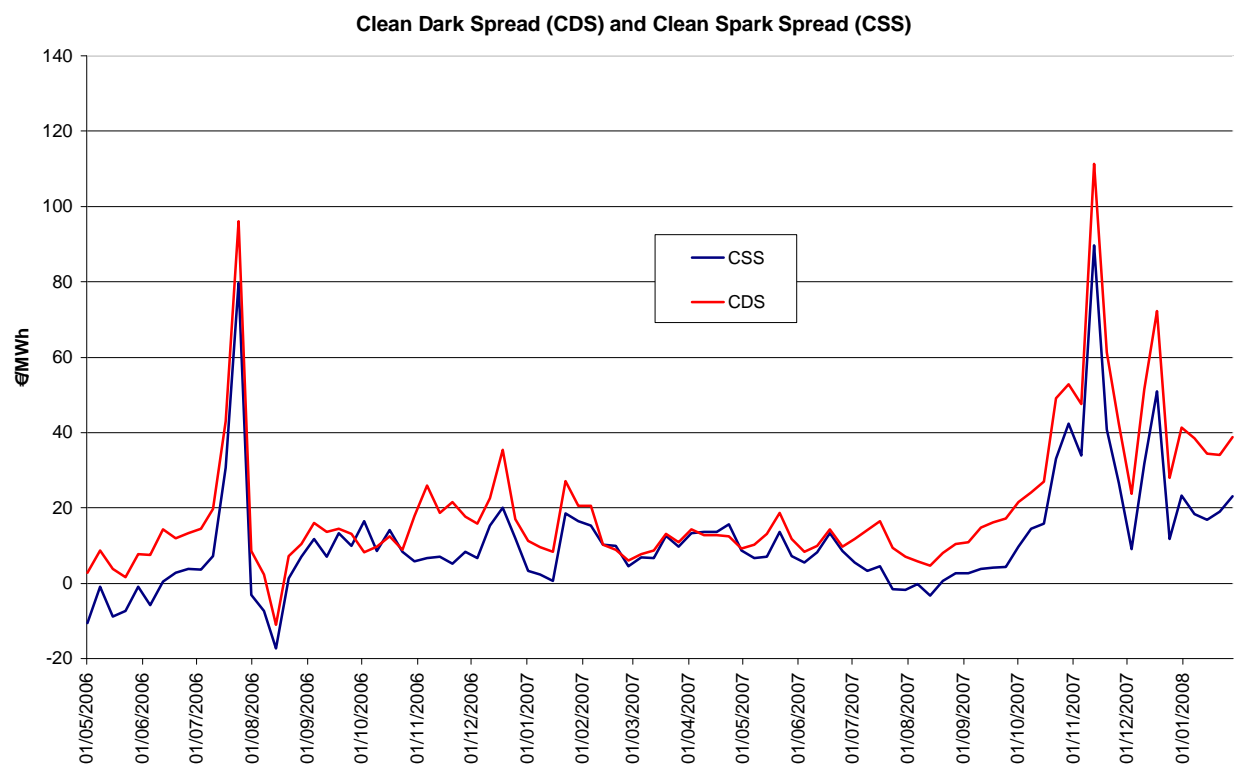

Figure 1: Clean dark spread and clean spark spread, May-2006 to Jan-2008. 


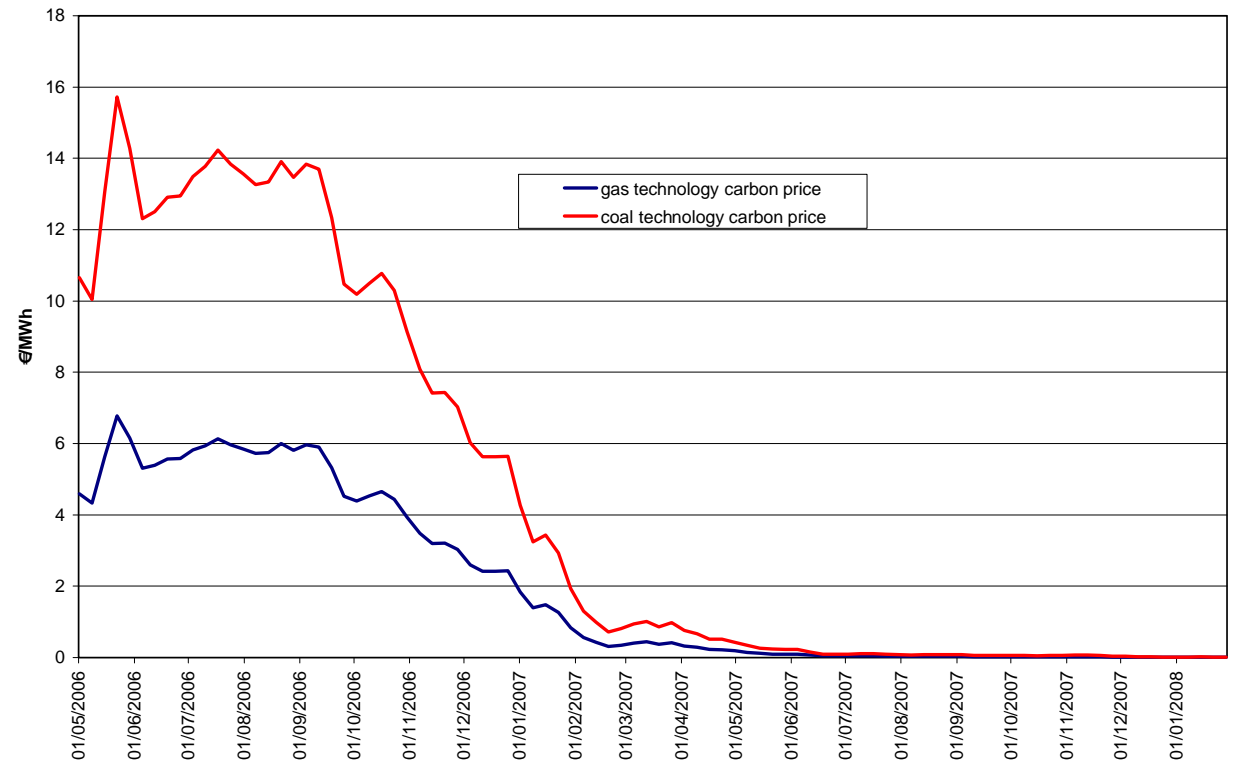

Figure 2: Carbon price $(€ / \mathrm{MWh})$ for gas- and coal-based technologies (assuming net thermal efficiencies $E_{G}=55 \%$ and $E_{C}=40 \%$ ). 
Partial Autocorrelation Function

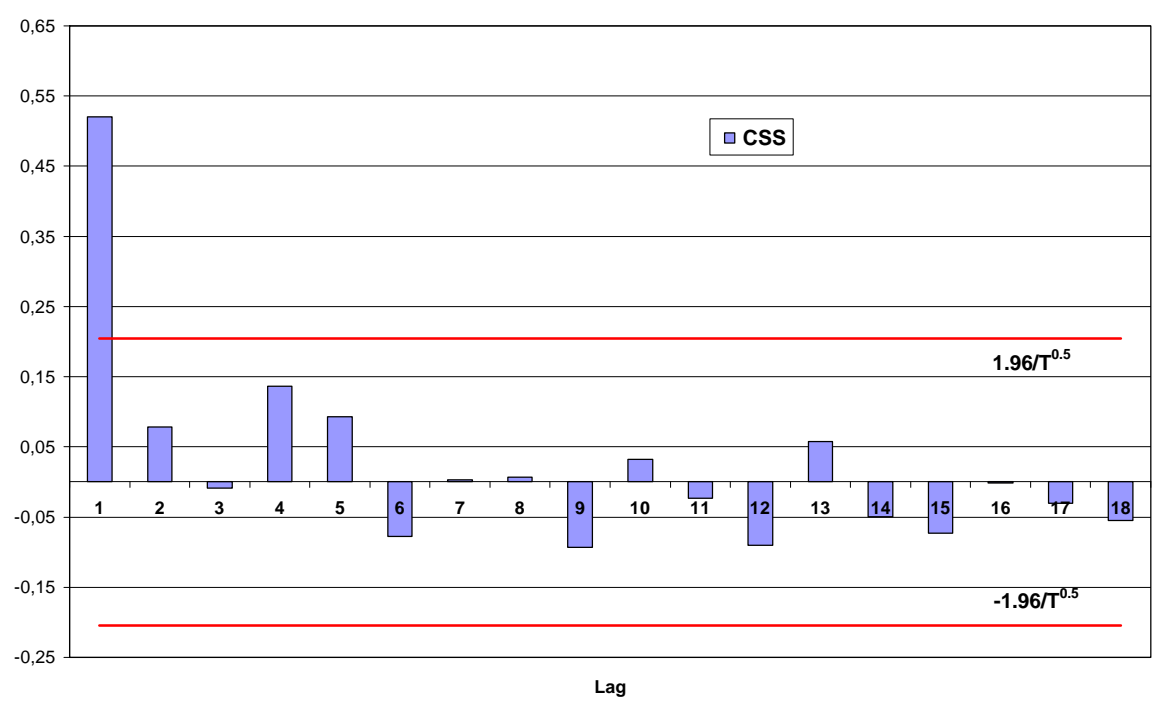

Figure 3: Partial autocorrelation function of the CSS series.

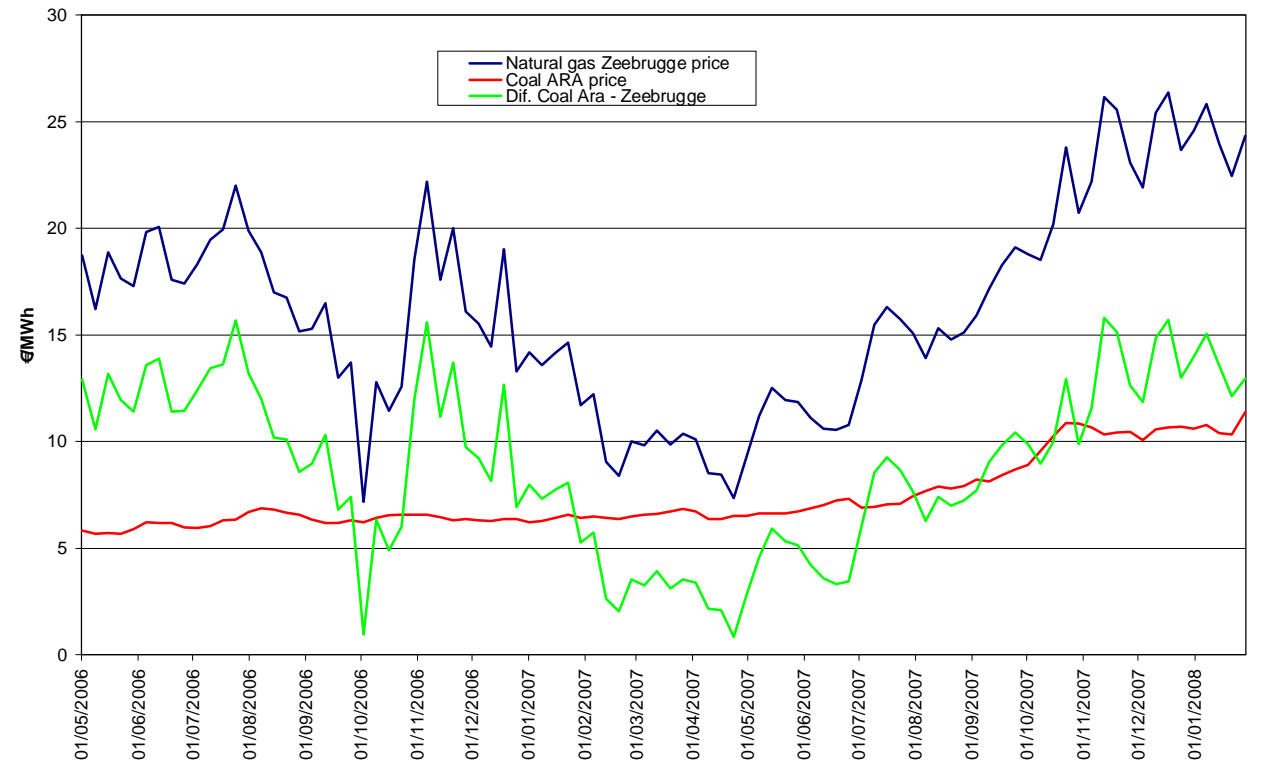

Figure 4: Natural gas (Zeebrugge) price, coal ARA price, and fuel price gap. 
Real and Estimated CSS

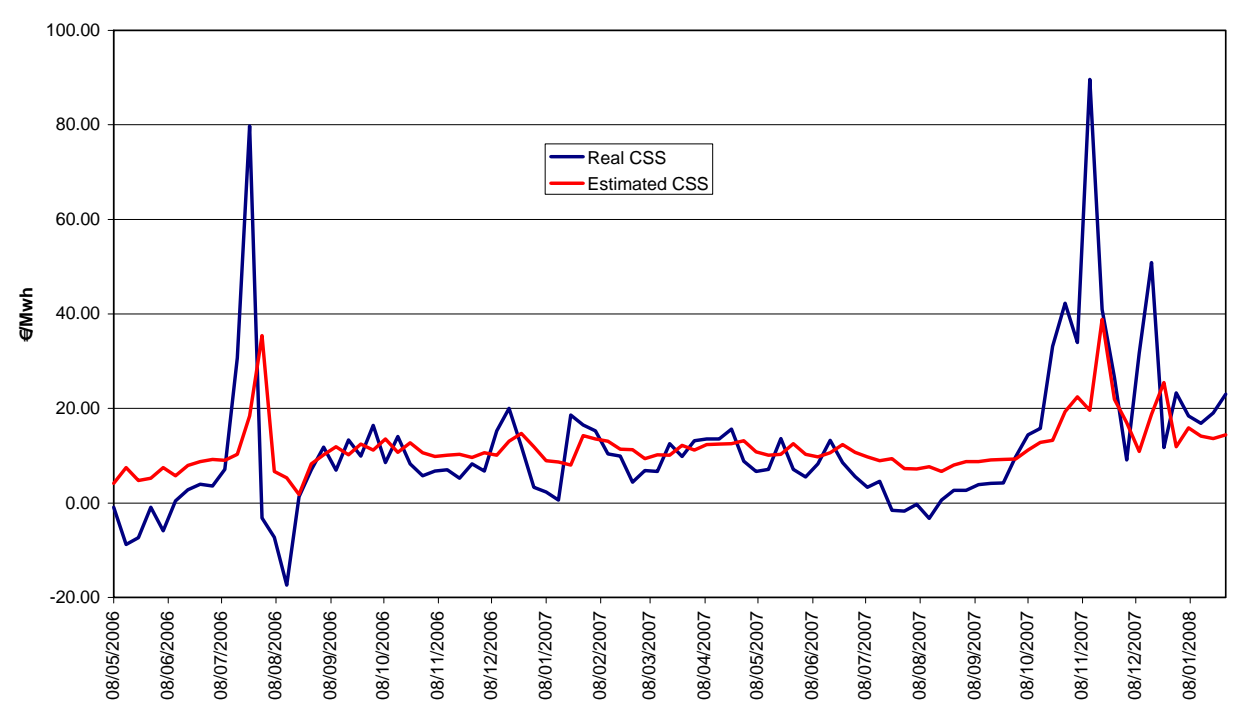

Figure 5: Actual and estimated CSS series. 


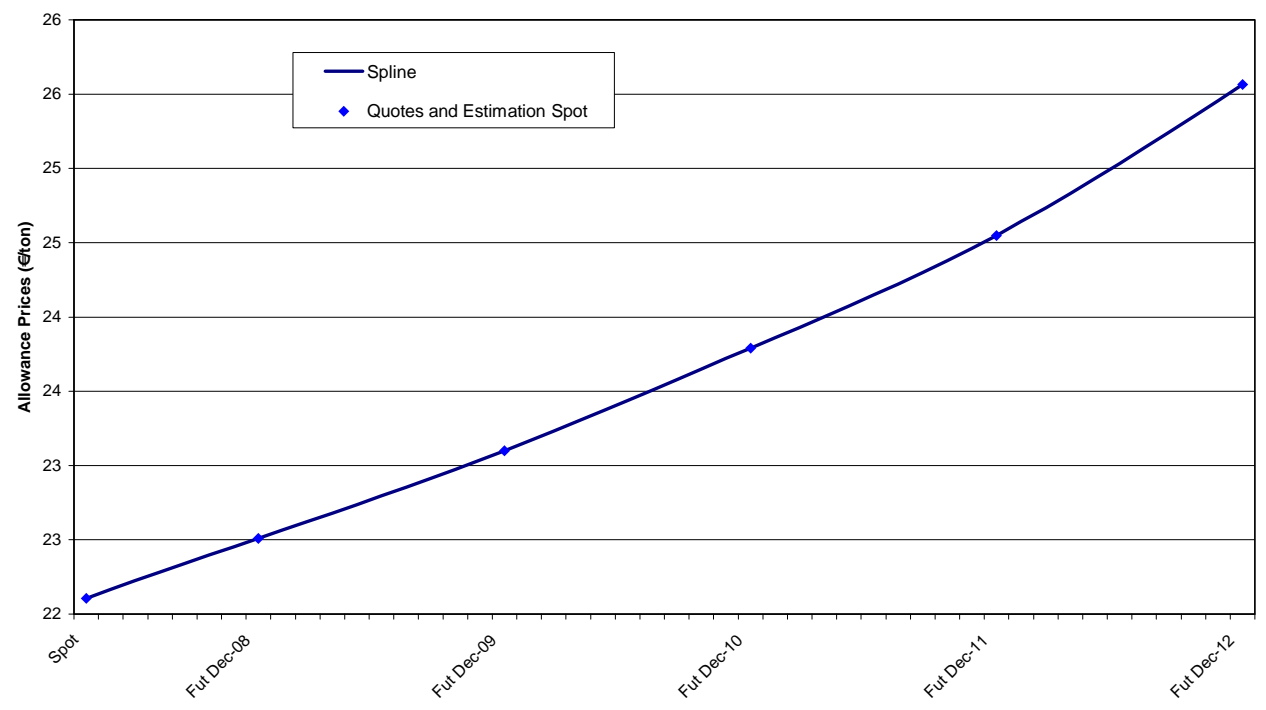

Figure 6: Spline-based estimation of spot price from futures prices (March 26th 2008). 


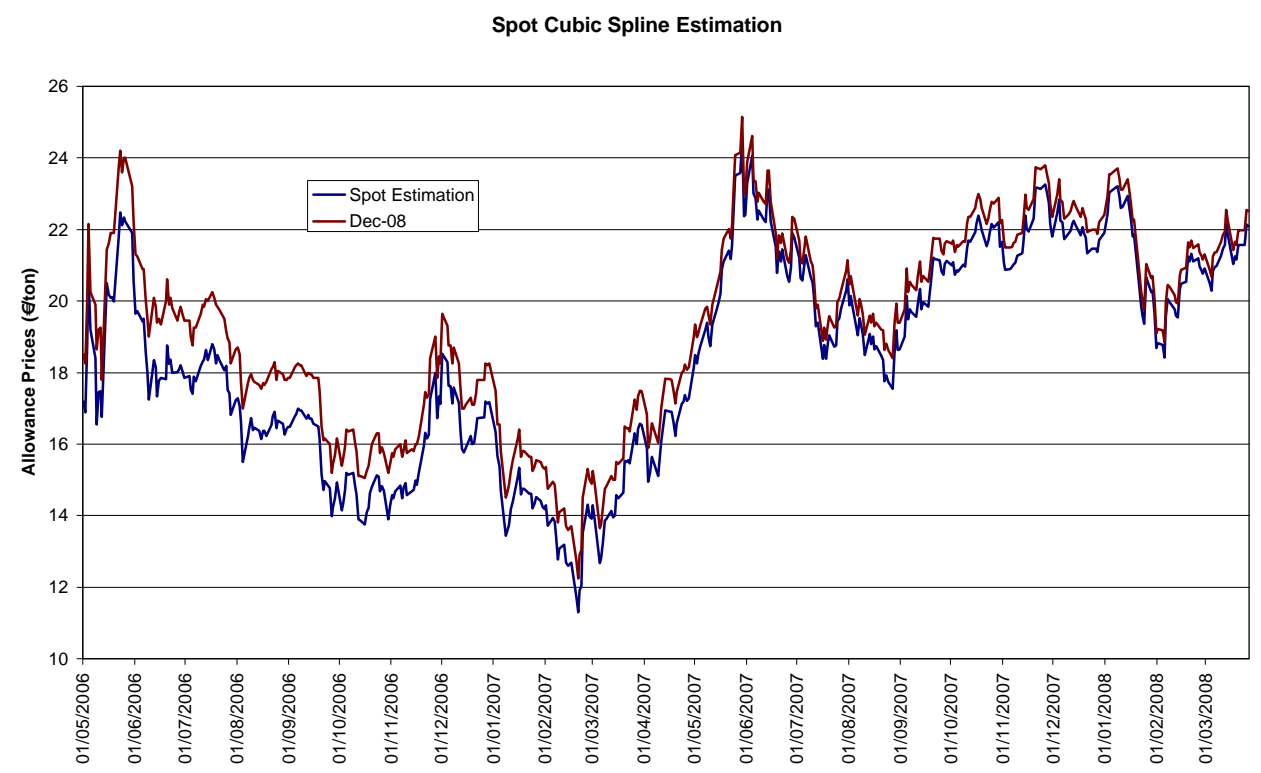

Figure 7: Spot estimates and nearby futures prices.

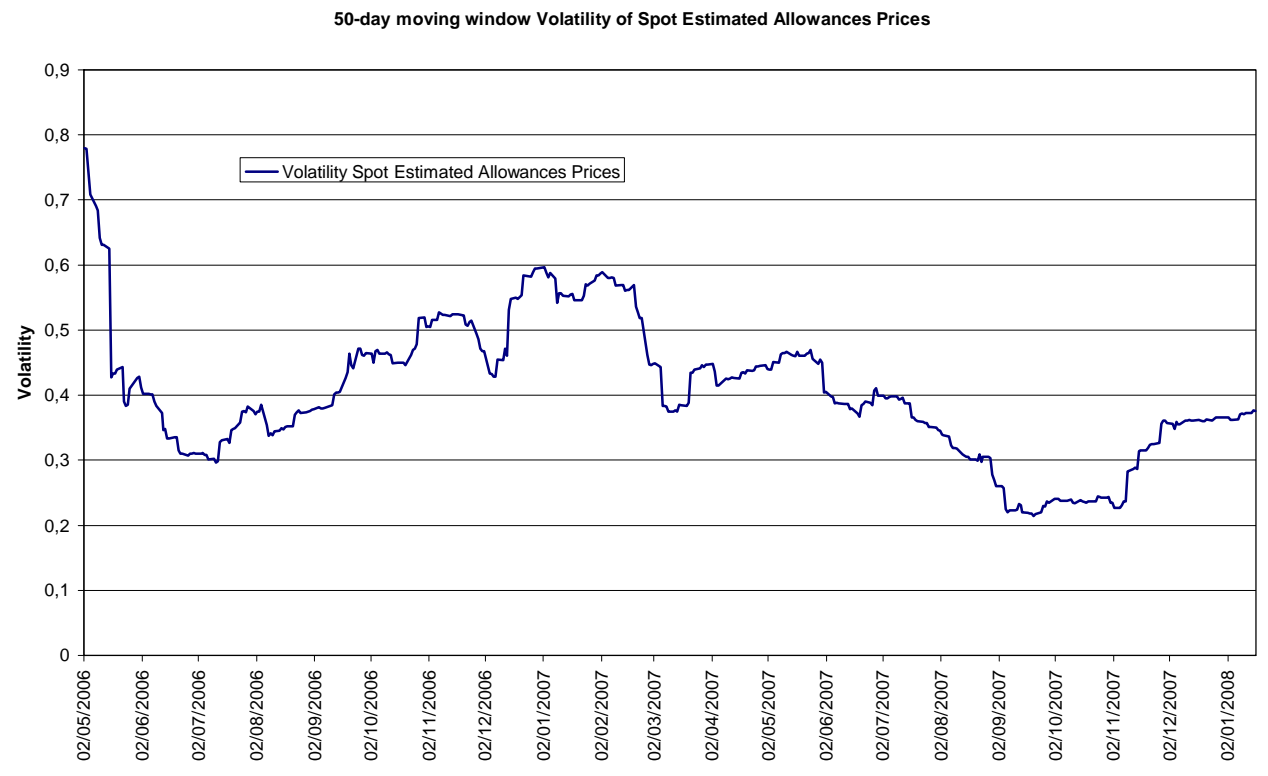

Figure 8: Volatility of spot allowance price over 50-days moving windows. 


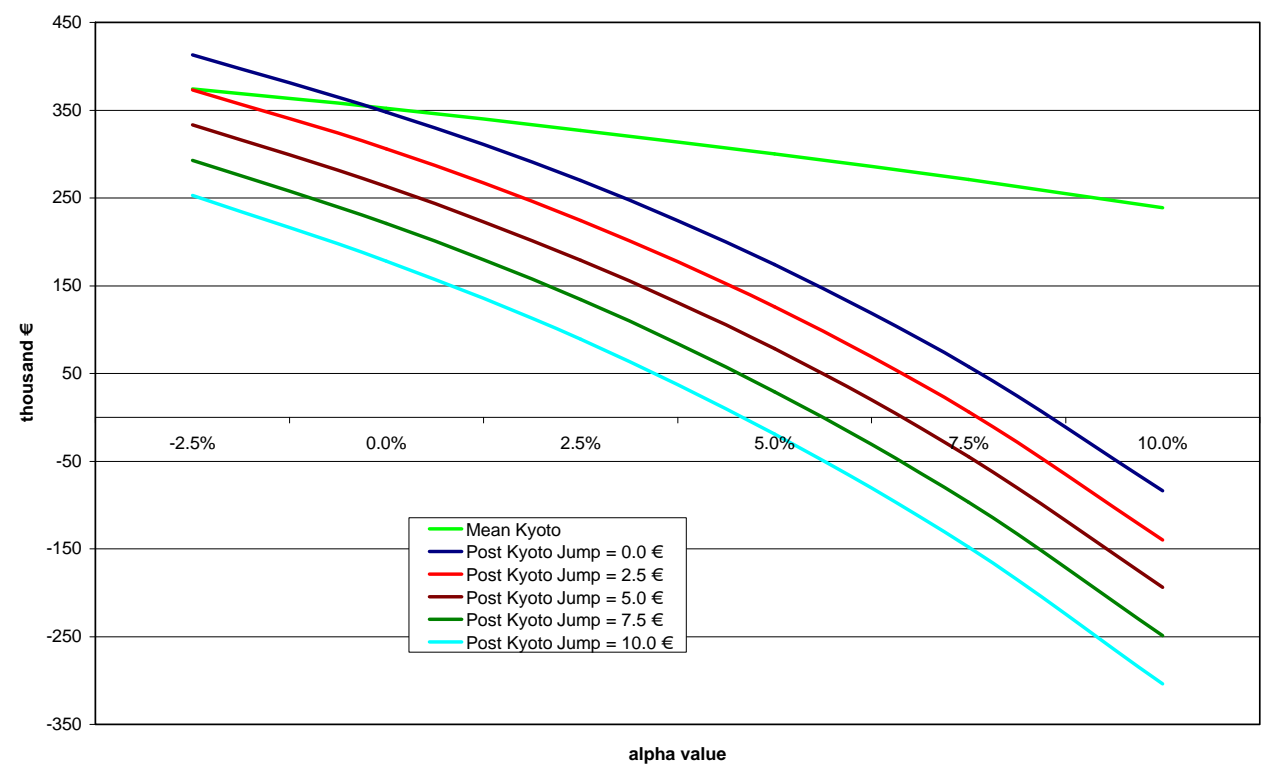

Figure 9: Expected earnings in the Kyoto period (2008-12) and beyond (201317). 


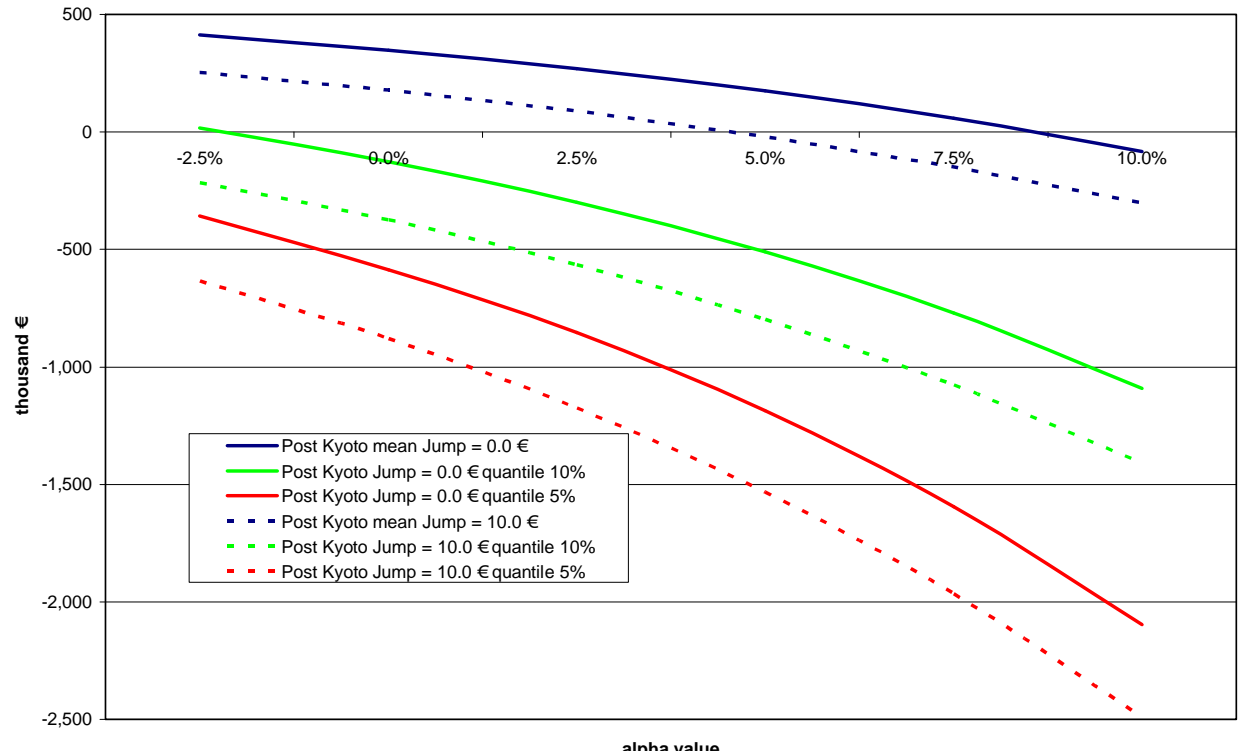

Figure 10: Earnings at Risk beyond Kyoto (2013-17): 5\% and 10\% percentiles. 